\title{
Morphology of Organic Matrix of Human Enamel
}

\author{
V.M. Dusevich ${ }^{1}$, J. McGuire ${ }^{1}$, J.P. Gorski ${ }^{1}$, Y. Wang ${ }^{1}$, M.P. Walker ${ }^{1}$ \\ ${ }^{1}$ School of Dentistry, University of Missouri - Kansas City, Kansas City, MO 64108
}

Adult human enamel is a highly mineralized tissue and contains just about $2 \%$ protein by volume, with the rest mostly of a hydroxyapatite [1]. Recently it was shown that protein distribution in enamel is not uniform: insoluble proteins concentrate in a band of 100-400 microns width which extends from dentin into enamel body and form an organic matrix [2].

Dentin - enamel junction (DEJ) has a complex organization and plays an important role in structural strength of a tooth; it transfers applied loads from enamel to dentin and arrest cracks initiating in enamel. Enamel organic matrix may strengthen DEJ by stabilizing inner layer of enamel and increasing its bonding to dentin [3]. However, properties and morphology of organic matrix are still insufficiently known. In this work morphology of organic matrix was studied by means of electron microscopy (SEM and TEM). Specimens were carefully demineralized (not to disturb delicate matrix), fixed with glutaraldehyde, dehydrated and some of them (for TEM study) were embedded in a resin.

The important morphological feature of an organic matrix is that it represents a fine meshwork of small fibers of about $20 \mathrm{~nm}$ in diameter (Fig. $1 \mathrm{a}, \mathrm{b}$ ). There is a strong indication that these are fibers of type VII collagen which can complex with other collagens and may contribute to the structural resilience of enamel and play a role in bonding enamel to dentin [4].

Crystals of hydroxyapatite in human enamel are long but narrow (20-80 nm in cross section); they are organized in parallel bundles, so called "enamel rods," of about 4-8 microns in diameter. Morphologically organic matrix is following the shape of these rods. Though organic matter is distributed throughout rod, most often it is concentrated in sheath regions (Fig. $1 \mathrm{c}, \mathrm{d}$ ), and less frequently sheath regions are depleted of organic matter (Fig 1 e). Organic matrix has highly variable structure and changes its morphology even in the same tooth frequently. Figure if shows a region in which sheath regions are changing from enriched by organic matter (on the right) to depleted (on the left).

Utilization of charge contrast in ESEM [5] provides another way of observation of organic matrix. Areas of enamel enriched by organic matter are darker. Figure 1g presents regions of enamel where organic matrix concentrated on rods boundaries, and Figure 1 h shows regions where matrix was concentrated in rods bodies.

\section{References}

[1] D.F. Poole, N.W. Johnson, Arch Oral Biol. 12(12) (1967) p. 1621-34.

[2] V. Dusevich et al, Arch Oral Biol, 57(12) (2012), p. 1585-94.

[3] J. McGuire et al, Connective Tissue Research, 55 (S1) (2014), p. 33-37.

[4] J. McGuire et al, Bone, 63(2014), p. 29-35.

[5] Griffin, B.J. (1997) Microscopy and Microanalysis, 3 (S2) (1997) 1197-1198.

[6] This research was supported by NIH/NIDCR grant R01DE021462. 

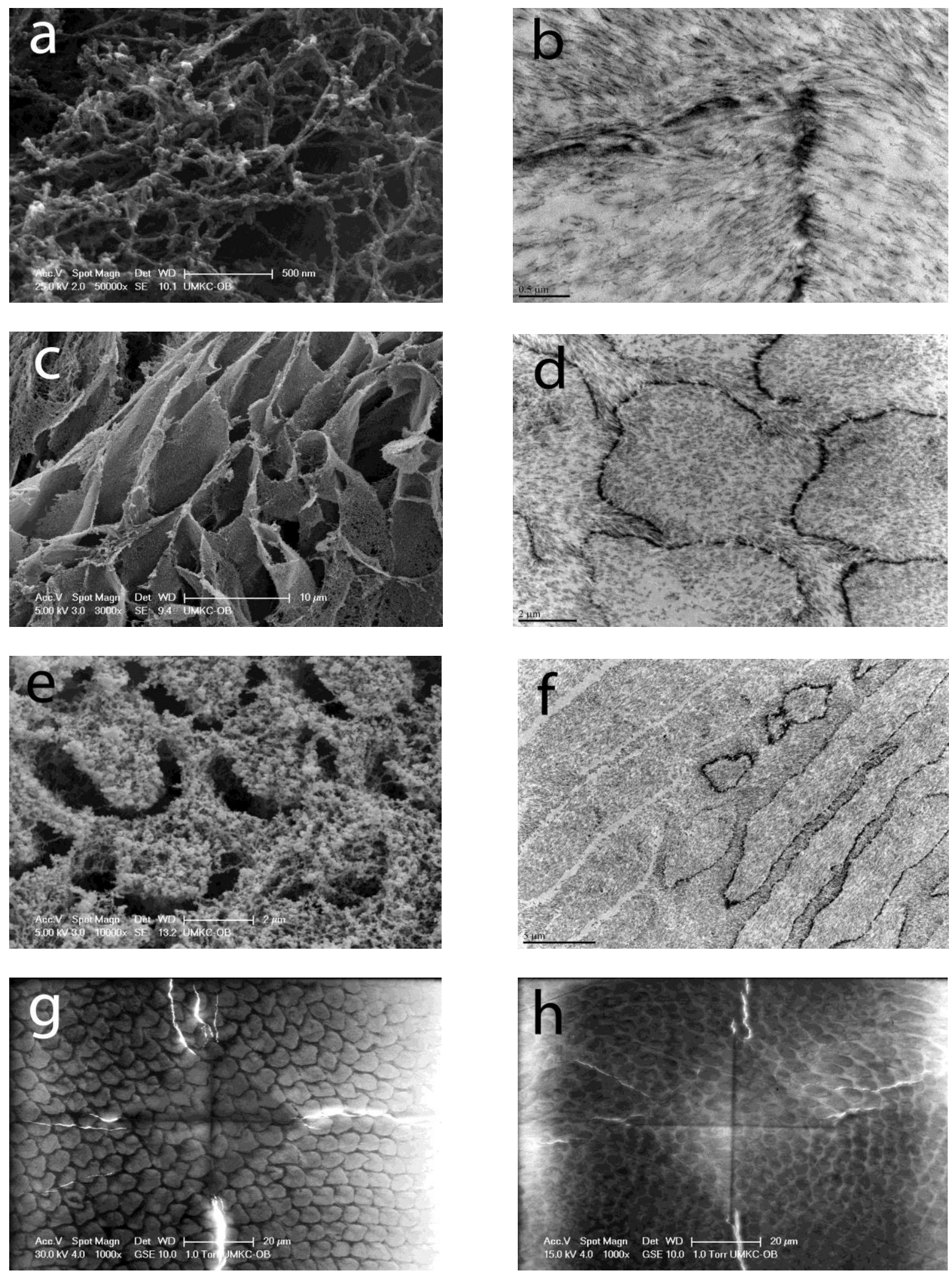

Figure 1. Elements of an organic matrix of human enamel. a) SEM, bar $200 \mathrm{~nm}$; b) TEM, bar $0.5 \mathrm{um}$; c) SEM, bar 10 um; d) TEM, bar 2 um; e) SEM, bar 2 um; f) TEM, bar 5 um; g) ESEM, bar 20 um; h) ESEM, bar 20 um 